Journal of Applied Pharmaceutical Science Vol. 6 (10), pp. 195-199, October, 2016

Available online at http://www.japsonline.com

DOI: $10.7324 /$ JAPS.2016.601027

ISSN 2231-3354 (cC) BY-NC-SA

\title{
Eucerin impedes transdermal insulin delivery by sonophoresis in rats
}

\author{
Nasrollah Jabbari ${ }^{*}$, Peyman Mikaili ${ }^{2}$, Mohammad Hossein Asghari ${ }^{3}$, Hassan Ahmadin ${ }^{4}$ \\ ${ }^{1}$ Solid Tumor Research center, Department of Medical Physics and Imaging, Urmia University of Medical Sciences, Urmia, Iran. \\ ${ }^{2}$ Department of Pharmacology, Urmia University of Medical Sciences, Urmia, Iran. \\ ${ }^{3}$ Department of Pharmacology, Faculty of Medicine, Babol University of Medical Sciences, Babol, Iran. \\ ${ }^{4}$ Department of Biomedical Engineering, Urmia University of Medical Sciences, Urmia, Iran.
}

\begin{tabular}{l} 
ARTICLE INFO \\
\hline Article history: \\
Received on: $05 / 07 / 2016$ \\
Revised on: 29/07/2016 \\
Accepted on: 17/09/2016 \\
Available online: $29 / 10 / 2016$ \\
\hline Key words: \\
Ointment insulin, rat, \\
sonophoresis, transdermal \\
drug delivery, low-frequency \\
ultrasound.
\end{tabular}

\begin{abstract}
The present study was designed to evaluate the effect of low-frequency ultrasound on transdermal delivery of insulin as an ointment. This was conducted in the proceeding of a previously published paper by the authors. Forty-two Wistar rats were randomly divided into 7 groups including one control and six experimental groups with six rats in each group. An ointment of insulin was prepared and applied (alone and with low-frequency ultrasound) on the skin of animals. In all the groups, blood samples were collected from the tail vein of the animals every 15 minutes of the whole 90-minute experimental period. The changes of blood glucose levels with time were assessed in the groups. The results of this study showed that the exposure of the insulin ointment to low-frequency ultrasound for a period of 60 minutes significantly decreased its potency in lowering the blood glucose levels. We conclude that eucerin impedes transdermal insulin delivery by sonophoresis in rats. We suggest that the lowering effect of ultrasound on the penetration ability of insulin is probably due to its effect on the specific molecular structure formed between the insulin and the ointment base after its preparation. However, further studies are needed in order to more precisely identify the involving mechanisms.
\end{abstract}

\section{INTRODUCTION}

Diabetes mellitus is an endocrine disorder in which the cells of the body are unable to properly metabolize sugar due to a total or relative lack of insulin (Khan, 2012). It is one of the main problems of health care systems of most societies. The total number of people with diabetes worldwide was estimated to be 131 million in 2000; it is projected to increase to 366 million by 2030 (Wild et al., 2004). At present, subcutaneous injection of insulin is the most common treatment method in patients with diabetes mellitus type 1 which affects their quality of life due to its invasive nature (Li et al., 2014; Hilliard et al., 2013). However, several obstacles such as day-to-day variability in insulin requirements and slow and variable absorption of insulin from the subcutaneous site of injection make it difficult for type 1 diabetic patients to maintain long-term near-normoglycemia

\footnotetext{
* Corresponding Author

Nasrollah Jabbari, Solid Tumor Research center, Department of Medical Physics and Imaging, Urmia University of Medical Sciences, Urmia, Iran.Email: njabbarimp @ gmail.com
}

(Lepore et al., 2000). Transdermal drug delivery (TDD) is considered as a potential painless alternative for the delivery of drugs such as insulin to diabetic patients during recent years (Schoellhammer et al., 2014). The main obstacle this method of drug delivery is dealing with is the limited skin permeability mainly due to the specific structure of the outer layer of the skin (stratum corneum) (Mohammadi-Samani et al., 2014). The development of a sophisticated new technique to overcome the skin permeability obstacle has challenged the researches to develop new delivery methods (Khafagy et al., 2007). In the last years, many researchers have been focusing on different ways of enhancing skin permeability such as ionophoresis (Naik et al., 2000), ultrasound-mediated transdermal drug delivery (Lavon et al., 2004), use of liposomes (Barry, 2001), etc. A great range of chemical absorption enhancers (CAEs) such as surfactants, fatty acids, iodine, short synthetic peptide and trypsin have been also used for this purpose (Chen et al., 2009; Sintov et al., 2007). However, some disadvantages including skin irritation and inability to deliver large molecules, have largely limited the use of these chemicals. 
Therefore, safer ways of enhancing transdermal drug absorption such as sonophoresis (application of ultrasound to enhance transdermal drug delivery) have been greatly taken into consideration during the last two decades (Karande et al., 2009). Irradiation of the skin with ultrasound followed by the application of insulin has indicated that the transdermal transport of insulin occurs mainly during sonophoresis (Polat et al., 2010).

Previous studies have shown that low-frequency ultrasound $(20-100 \mathrm{kHz})$ is effective in the enhancement of transdermal insulin delivery (Boucaud et al., 2002; Baris et al., 2010; Jabbari et al., 2015). Although the effective principle of sonophoresis is not well-understood, it has been suggested to be the result of cavitations in the skin (Smith, 2007; Park et al., 2007). It has to be said that the parameters and surrounding physiochemical conditions under which ultrasound is applied have the most important role in the effects observed with the application of sonophoresis.

Overall, it has been concluded in the literature that the transdermal insulin delivery can be enhanced with $40 \mathrm{kHz}$ lowfrequency ultrasound (Smith, 2007; Park et al., 2007) and with insulin ointment alone (Jabbari et al., 2015), but the enhancement effects of low-frequency ultrasound with insulin ointment are not clear and were evaluated in this study for the first time.

The aim of this study was to evaluate the effect of low-frequency ultrasound on transdermal delivery of insulin as an ointment and to clarify the target structure that is affected by ultrasound at different conditions of sonophoresis.

\section{MATERIAL AND METHODS}

\section{Ointment preparation}

The insulin ointment was prepared using pure human insulin (Humulin ${ }^{\circledR}$ R, rDNA U-100, Indianapolis, IN) and eucerin at room temperature. Eucerin is commonly used for therapeutic and cosmetic purposes (Sexton, 1940). It is among one of five major types of substances used in preparation of ointments (Nielloud et al., 2007). Desirable pharmacological properties of eucerin such as good drug release potential and hydrophilic capability (Jabbari et al., 2015) have made it a good choice to be used in the preparation of oil-based ointments. 4, 8, 9.4 and 10.2 $\mathrm{ml}$ of regular insulin were calculated each to be added to $2 \mathrm{~g}$ of eucerin in order to reach $0.69 \%, 1.36 \%, 1.6 \%$, and $1.73 \%$ concentrations of insulin ointment, respectively. The mentioned concentrations were calculated as weight/weight percentages (Sintov et al., 2007). To prepare the ointment, calculated amounts of insulin for each treatment group was gradually added to $2 \mathrm{~g}$ of eucerin while the mixture was being agitated. At the times that the mixture lost its consistency, the addition of insulin was seized for a while until it could regain its consistency so that the remaining amount of insulin could be added. Under these conditions, insulin can be readily mixed into eucerin. Finally, the ointment was stored at $4{ }^{\circ} \mathrm{C}$ until commencement of the experiment. Our previous study (Jabbari et al., 2015) showed that the blood glucose lowering activity of the $1.73 \%$ insulin ointment was almost equal to that of the injectable insulin. Thus, in this study, the $1.73 \%$ concentration was selected as the insulin ointment.

\section{Ultrasound transducer array}

In the previous study, we developed equipment for transdermal insulin delivery, so that the geometrical and physical characteristics of this equipment were briefly described in the literature (Jabbari et al., 2015). Ultrasound was produced by an air ultrasonic ceramic transducer (SQ-40-T-10B) composed of a piezo ceramic disc which converts electric energy into mechanical energy. The diameter of each ultrasonic sensor was $9.8 \mathrm{~mm}$ and the resonant frequency of them was $40 \pm 1.0 \mathrm{kHz}$. Figure 2 shows an array air ultrasonic ceramic transducer used in this study. The array includes three ultrasonic probes connected in parallel.

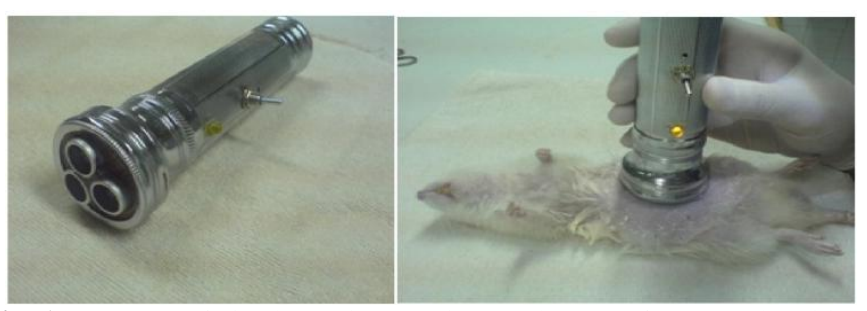

Fig. 1: An array of air ultrasonic ceramic transducer (Left), a rat placed in a dorsal decubitus position with the transducer array attached (Right).

\section{Animal experiments}

All procedures described in this study involving animal experiments were approved by Ethical Committee of Urmia University of Medical Sciences (approval number 56-06). Forty two male Wistar rats (237-380 g) were randomly divided into 7 groups each with 6 animals. The number of animals for control and experimental groups was determined based on the pertinent literature (Park et al., 2008; Jabbari et al., 2015). All the animals were fed on standard chow diet and were kept in a stress-free condition two weeks before beginning of the experiment. A combination of ketamin hydrochloride $(60 \mathrm{mg} / \mathrm{kg})$ and xylazine hydrochloride $(10 \mathrm{mg} / \mathrm{kg})$ was intra-peritoneally injected in all animals to anaesthetize them (Ketamine 10\%+Xylazine 2\%). Xylazine was used for general anesthesia and also inducing a temporary but sustained hyperglycemia in the animals during the experimental period. The first group remained untreated as the control group, the second and the third groups received insulin ointment and subcutaneous insulin injection $(0.25 \mathrm{U} / \mathrm{Kg})$, respectively and the other groups were treated with ultrasound under the following conditions: received ultrasound after the ointment was applied on the skin (group 4); insulin was delivered subcutaneously after it was exposed to ultrasound (group 5); the ointment was applied on the skin after pre-irradiation of eucerin with ultrasound (group 6); the ointment was treated with ultrasound before its application on the skin (group 7). In order for the transdermal application of insulin, the abdominal area of each rat was shaved with dimensions of $5 \times 5 \mathrm{~cm}^{2}$ and the abdominal surface was carefully cleaned to remove any remaining hair. In the groups with ultrasound exposure, following the administration of 
the insulin ointment, the designed ultrasonic array was placed on the skin perpendicularly and then ultrasound waves with $40 \mathrm{kHz}$ frequency were applied for 60 minutes.

The ultrasound was applied in pulse mode to avoid any undesired damage to the skin as a result of the heat produced. Examination of the rat's skin was performed at the end of ultrasonic irradiation and insulin ointment administration to look for visible lesions such as rash, redness, and swelling on the skin surface.

Blood samples were collected from the tail vein of the animals once before anesthesia to confirm the uniformity of the glucose levels in all the animals and for the second time after anesthesia and prior to the application of the ointment and the ultrasound to obtain a baseline glucose (sugar) level. Additional blood samples were taken every 15 minutes of the whole 90minute experiment. An ACCU-CHEK ${ }^{\mathrm{TM}}$ blood glucose monitoring system (Roche Diagnostics Co., Indianapolis) was used to test the blood samples. Each sample was tested at least twice to confirm the accuracy of the readings. In order for comparing the changes in blood sugar levels, the data were corrected by subtracting the baseline sugar for each animal from each blood sugar level at any time.

Statistical analysis was performed using SPSS software (ver. 21), by SPSS Inc. (IBM corporation, Armonk, USA). Oneway ANOVA statistical test was used to confirm the uniformity of the weight in all the animals and blood sugar levels before anesthesia. Moreover, the statistical multiple comparisons (Dunnett's and Tukey's) tests were used to compare the difference between glucose levels in the studied groups after anesthesia. The significance level was selected as 0.05 for all the statistical tests. The blood sugar versus time data were pooled for each group and analyzed as its mean and standard deviation (SD).

\section{RESULTS}

The objective of this study was to evaluate the effect of low-frequency ultrasound on transdermal delivery of insulin as an ointment. Visual examination of the rats' skins at the end of ultrasonic exposimetry and insulin ointment administration did not show any damage or significant change to the skin. Table 1 shows the changes of blood glucose levels with time in different groups.
Performing One-way ANOVA statistical test between seven studied groups showed no significant difference between the weight of the rats $(\mathrm{p}=0.103)$ and blood glucose concentration before anesthesia $(p=0.476)$. The blood glucose concentration before anesthesia between the study groups was within a range of 101 to $118 \mathrm{mg} / \mathrm{dl}$. Following the administration of xylazine, the initial blood glucose levels increased to $193.85 \pm 13.78 \mathrm{mg} / \mathrm{dl}$ (mean \pm SD) after anesthesia, which was called the baseline glucose level. The statistical multiple comparisons (Dunnett) test showed that there was no significant difference between the glucose levels in the control and treatment groups after anesthesia ( $p>0.05$ ). Multiple comparisons (Dunnett) test was also performed on the mean blood sugar levels between the control group and the treatment groups at different follow-up time points during the experiment period (Table 2). The blood glucose levels of all the animals were graphed and recorded as the mean \pm SD of each group every 15 minutes of the whole 90 minute experimental period (Figure 2). Due to the anesthesia with xylazine, the initial blood glucose levels were higher than that of normal rats before anesthesia. The blood glucose levels in the third, fifth and sixth groups were significantly different from those of the control group 15, 30 and 45 minutes after anesthesia. In addition to these three groups, the glucose levels in the second group significantly differed from the control group 60 minutes after anesthesia. Group seven was the only group that showed no significant difference with the control group even after 90 minutes $(\mathrm{P}>0.05)$. The glucose levels in the second group (sole insulin ointment) was significantly lower than those in the control group $(\mathrm{P}<0.05)$ indicating that the ointment is effective in lowering the blood glucose levels in hyperglycemic rats.

Exposure of the skin to $40 \mathrm{kHz}$ ultrasound along with treatment of the skin with the ointment (group 4) significantly delayed and reduced the blood glucose lowering activity of the ointment in a way that after treatment with both of the ointment and the ultrasound, the glucose levels started to fall after 75 minutes, implying the disruptive effect of the ultrasound on insulin efficiency in lowering blood glucose. This effect was also observed even after pre-irradiation of the ointment with ultrasound before its administration on the skin (group 7) vanished its ability to decrease blood glucose levels, in comparison with the control group $(\mathrm{P}<0.05)$.

Table 1: Changes of blood glucose levels with time in different groups.

\begin{tabular}{|c|c|c|c|c|c|c|c|c|}
\hline \multirow{3}{*}{ Groups } & \multicolumn{8}{|c|}{$\underline{\text { Mean } \pm \text { SD of blood glucose levels (mg/dl) }}$} \\
\hline & \multicolumn{8}{|c|}{ After anesthesia } \\
\hline & Before anesthesia & ( $\mathrm{Min}$ & 15 Min & 30 Min & 45 Min & 60 Min & 75 Min & 90 Min \\
\hline Group 1 & $109.94 \pm 7.52$ & $183.21 \pm 13.21$ & $242.67 \pm 17.95$ & $253.66 \pm 21.42$ & $280.33 \pm 19.13$ & $285.33 \pm 22.03$ & $286.33 \pm 18.12$ & $287.26 \pm 16.43$ \\
\hline Group 2 & $115.02 \pm 4.36$ & $220.67 \pm 12.34$ & $260.68 \pm 14.58$ & $256.48 \pm 16.85$ & $232.56 \pm 13.23$ & $246.43 \pm 13.52$ & $177.24 \pm 12.68$ & $164.64 \pm 18.51$ \\
\hline Group 3 & $106.33 \pm 8.50$ & $200.76 \pm 14.28$ & $181 \pm 13.76$ & $123.54 \pm 11.07$ & $89.68 \pm 12.38$ & $80.56 \pm 9.63$ & $71.33 \pm 8.40$ & $66.43 \pm 6.18$ \\
\hline Group 4 & $106.14 \pm 5.56$ & $207.66 \pm 16.46$ & $222.18 \pm 18.21$ & $230.33 \pm 17.65$ & $236.33 \pm 16.28$ & $227.42 \pm 17.21$ & $217.64 \pm 18.32$ & $211.34 \pm 16.57$ \\
\hline Group 5 & $110.33 \pm 6.43$ & $194.46 \pm 17.33$ & $146.67 \pm 15.12$ & $105.33 \pm 12.31$ & $85.33 \pm 9.12$ & $78.64 \pm 7.41$ & $68.54 \pm 5.24$ & $58.67 \pm 5.12$ \\
\hline Group 6 & $107.05 \pm 7.21$ & $159.33 \pm 16.45$ & $167.33 \pm 17.56$ & $161.67 \pm 18.41$ & $159.45 \pm 14.65$ & $149.66 \pm 15.42$ & $141.87 \pm 16.22$ & $130.67 \pm 14.12$ \\
\hline Group 7 & $104.67 \pm 2.52$ & $190.43 \pm 19.52$ & $205.62 \pm 21.36$ & $219.34 \pm 20.45$ & $245.81 \pm 21.84$ & $238.33 \pm 19.44$ & $234.55 \pm 21.35$ & $229.16 \pm 22.63$ \\
\hline
\end{tabular}


Exposure of insulin to ultrasound before its subcutaneous delivery (group 5) did not have any effect on its blood glucose decreasing activity. In other words, blood glucose lowering capability of the ointment was still maintained even after the exposure of eucerin to ultrasound. This indicated that ultrasound had no effect on the eucerin structure so that the ointment activity was still present after exposure to ultrasound.

Table 2: The results of multiple comparisons (Dunnett's) test of the mean blood sugar levels in the studied groups of rats at different times of the experiment period.

\begin{tabular}{|c|c|c|}
\hline Time (Min) & Multiple Comparisons (Dunnett's) & P-Value \\
\hline \multirow{6}{*}{15} & $\mathrm{G}_{2}-\mathrm{G}_{1}$ & 0.019 \\
\hline & $\mathrm{G}_{3}-\mathrm{G}_{1}$ & 0.025 \\
\hline & $\mathrm{G}_{4}-\mathrm{G}_{1}$ & 0.852 \\
\hline & $\mathrm{G}_{5}-\mathrm{G}_{1}$ & 0.001 \\
\hline & $\mathrm{G}_{6}-\mathrm{G}_{1}$ & 0.002 \\
\hline & $\mathrm{G}_{7}-\mathrm{G}_{1}$ & 0.257 \\
\hline \multirow{6}{*}{30} & $\mathrm{G}_{2}-\mathrm{G}_{1}$ & 0.001 \\
\hline & $\mathrm{G}_{3}-\mathrm{G}_{1}$ & 0.001 \\
\hline & $\mathrm{G}_{4}-\mathrm{G}_{1}$ & 0.872 \\
\hline & $\mathrm{G}_{5}-\mathrm{G}_{1}$ & 0.001 \\
\hline & $\mathrm{G}_{6}-\mathrm{G}_{1}$ & 0.001 \\
\hline & $\mathrm{G}_{7}-\mathrm{G}_{1}$ & 0.508 \\
\hline \multirow{6}{*}{45} & $\mathrm{G}_{2}-\mathrm{G}_{1}$ & 0.001 \\
\hline & $\mathrm{G}_{3}-\mathrm{G}_{1}$ & 0.001 \\
\hline & $\mathrm{G}_{4}-\mathrm{G}_{1}$ & 0.340 \\
\hline & $\mathrm{G}_{5}-\mathrm{G}_{1}$ & 0.001 \\
\hline & $\mathrm{G}_{6}-\mathrm{G}_{1}$ & 0.001 \\
\hline & $\mathrm{G}_{7}-\mathrm{G}_{1}$ & 0.582 \\
\hline \multirow{6}{*}{60} & $\mathrm{G}_{2}-\mathrm{G}_{1}$ & 0.001 \\
\hline & $\mathrm{G}_{3}-\mathrm{G}_{1}$ & 0.001 \\
\hline & $\mathrm{G}_{4}-\mathrm{G}_{1}$ & 0.199 \\
\hline & $\mathrm{G}_{5}-\mathrm{G}_{1}$ & 0.001 \\
\hline & $\mathrm{G}_{6}-\mathrm{G}_{1}$ & 0.001 \\
\hline & $\mathrm{G}_{7}-\mathrm{G}_{1}$ & 0.405 \\
\hline \multirow{6}{*}{75} & $\mathrm{G}_{2}-\mathrm{G}_{1}$ & 0.001 \\
\hline & $\mathrm{G}_{3}-\mathrm{G}_{1}$ & 0.001 \\
\hline & $\mathrm{G}_{4}-\mathrm{G}_{1}$ & 0.154 \\
\hline & $\mathrm{G}_{5}-\mathrm{G}_{1}$ & 0.001 \\
\hline & $\mathrm{G}_{6}-\mathrm{G}_{1}$ & 0.001 \\
\hline & $\mathrm{G}_{7}-\mathrm{G}_{1}$ & 0.135 \\
\hline \multirow{6}{*}{90} & $\mathrm{G}_{2}-\mathrm{G}_{1}$ & 0.001 \\
\hline & $\mathrm{G}_{3}-\mathrm{G}_{1}$ & 0.001 \\
\hline & $\mathrm{G}_{4}-\mathrm{G}_{1}$ & 0.121 \\
\hline & $\mathrm{G}_{5}-\mathrm{G}_{1}$ & 0.001 \\
\hline & $\mathrm{G}_{6}-\mathrm{G}_{1}$ & 0.001 \\
\hline & $\mathrm{G}_{7}-\mathrm{G}_{1}$ & 0.068 \\
\hline
\end{tabular}

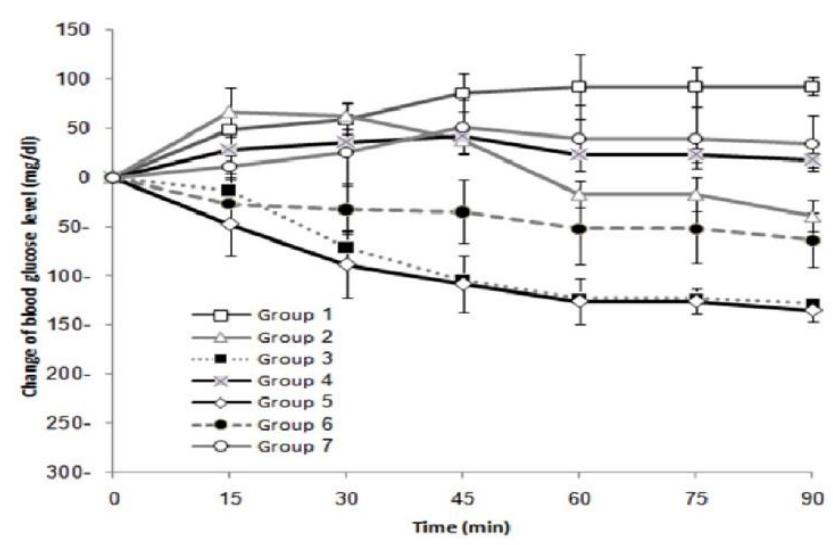

Fig. 2: Change of blood glucose levels during the experiment.

\section{DISCUSION}

The aim of this study was to evaluate the effect of lowfrequency ultrasound on transdermal delivery of insulin as an ointment. High frequency ultrasound (1-3 MHz) has been already investigated in different studies as a physical enhancer of transdermal drug delivery and it has been used as a therapeutic agent for over 40 years (Ferrara, 2008; Saroha et al., 2011).

Since this frequency range of ultrasound is not so potent when heating produced by the ultrasound equipment is minimized (Boucaud et al., 2001), the study of low-frequencies of ultrasound has been largely taken into consideration by many researchers during recent years (Park et al., 2008; Jabbari et al., 2015). An ultrasound range between $20 \mathrm{kHz}$ and $100 \mathrm{kHz}$ is normally considered as low-frequency (Park et al., 2008; Jabbari et al., 2015). This range of ultrasound is significantly more effective in the enhancement of transdermal delivery of various drugs (Lavon et al., 2004). For instance, low-frequency (20 kHz) ultrasound enhanced the transdermal transport of fentanyl and caffeine across human and rat skin (Boucaud et al., 2001). There are also many studies that show the efficiency of low-frequency ultrasound in the transdermal delivery of insulin as a non-invasive method (Park et al., 2008; Park et al., 2007; Ogura et al., 2008). In almost all the studies, the effect of sonophoresis depended, at least partially, on the ultrasound parameters including intensity, exposure time and conditions in which the ultrasound is applied (Boucaud et al., 2001; Ogura et al., 2008). Therefore, contradictory results have been observed in different studies as a consequence of different sonophoresis parameters and exposure conditions followed in each.

In the present study, we focused on the effects of lowfrequency $(40 \mathrm{kHz})$ ultrasound on the efficiency of transdermal insulin delivery using insulin ointment. In order to further evaluate the causative target structure affected by ultrasound during the ultrasound exposure, the ultrasound was applied at different conditions.

The results of this study show that the exposure of the insulin ointment to low-frequency ultrasound during a period of 60 minutes significantly decreases its potency in lowering the blood glucose levels. This is while separate exposure of the ointment compartments (insulin and eucerin) with ultrasound prior to administration of them as insulin ointment did not have any effect on their blood glucose lowering activity. No such inhibitory effect was observed even after exposure of the injectable form of insulin. Our data are in contrast with the results of many other studies that had previously shown the enhancing activity of ultrasound on transdermal drug delivery (Park et al., 2008; Jabbari et al., 2015; Ogura et al., 2008) with the suggested mechanism of sonophoresis. Here, we suggested a new probably mechanism by which ultrasound interferes transdermal transport of insulin which may be different from the sonophoresis mechanism previously suggested by some authors (Mitragotri et al., 2004; Park et al., 2007; Ogura et al., 2008). The reason for such a difference may be due to combinatorial construction of macromolecules during 
application of ultrasounic waves and/or after that. The results of this study showed that the lowering effect of ultrasound on the transdermal transportation of insulin is due to probable structural alterations of the ointment after its exposure to the ultrasound.

\section{CONCLUSION}

Our findings demonstrated the application of ultrasound $(40 \mathrm{kHz})$ on insulin ointment during a 60 -minute period, the insulin (as an ointment) will lose its ability to reduce blood glucose levels (group 7), while pre-irradiation of insulin (group 5) and eucerin (group 6) with ultrasound separately has no effect on the ointment activity. The target principle which is affected by the ultrasound during transdermal insulin delivery might be probably a specific molecular structure formed between different components of the insulin ointment (eucerin and insulin). Using low-frequency ultrasound for insulin delivery may be a promising method. However, in this study, we reported the incompatibility between insulin and eucerin (insulin ointment) affected by ultrasound beams. Although, eucerin is considered as a typical base for most ointments, the combination of insulin and eucerin (insulin ointment) irradiated by low-frequency ultrasound significantly inhibited the insulin to be absorbed $(\mathrm{p}<0.01)$. Further studies are needed in order to more precisely clarify effects produced by ultrasound during transdermal delivery of insulin and probable mechanisms involved as to whether it could be used for the enhancement of transdermal insulin delivery as a potential noninvasive rout. More studies are also required to specify the parameters under which the ultrasound should be applied to elicit enhancing effects of ultrasound during transdermal insulin delivery without possible undesired effects.

\section{ACKNOWLEDGEMENTS}

Financial support and sponsorship: We are thankful to Vice Chancellor for Research (VCR) of Urmia University of Medical Sciences for financial approval of this project.

Conflict of Interests: There are no conflicts of interest.

\section{REFERENCES}

Polat BE, Blankschtein D, Langer R. Low-Frequency Sonophoresis: Application to the Transdermal Delivery of Macromolecules and Hydrophilic Drugs. Expert Opin Drug Deliv, 2010; $7: 1415-32$.

Barry BW. Novel mechanisms and devices to enable successful transdermal drug delivery. Eur J Pharm Sci, 2001; 14:101-14.

Boucaud A, Machet L, Arbeille B, Machet MC, Sournac M, Mavon A, et al. In vitro study of low-frequency ultrasound-enhanced transdermal transport of fentanyl and caffeine across human and hairless rat skin. Int J Pharm, 2001; 228:69-77.

Schoellhammer CM, Blankschtein D, Langer R. Skin Permeabilization for Transdermal Drug Delivery: Recent Advances and Future Prospects. Expert Opin Drug Deliv, 2014; 11: 393-407.

Chen $\mathrm{H}$, Zhu $\mathrm{H}$, Zheng J, Mou D, Wan J, Zhang J, et al. Iontophoresis-driven penetration of nanovesicles through microneedleinduced skin microchannels for enhancing transdermal delivery of insulin. J Control Release, 2009; 139:63-72.
Ferrara KW. Driving delivery vehicles with ultrasound. Adv Drug Deliv Rev, 2008; 60:1097-102.

Jabbari N, Asghari MH, Ahmadian H, Mikaili P. Developing a Commercial Air Ultrasonic Ceramic Transducer to Transdermal Insulin Delivery. J Med Signals Sens, 2015; 5:117-22.

Jabbari N, Asghari MH, Mikail P. The efficiency of transdermal insulin delivery by using different concentrations of insulin ointment in hyperglycemic rats. Int J Res App Basic Med Sci, 2015; 1:20-6.

Hilliard ME, Mann KA, Peugh JL, Hood KK. How poorer quality of life in adolescence predicts subsequent type 1 diabetes management and control. Patient Educ Couns, 2013; 91:120-5.

Karande P, Mitragotri S. Enhancement of transdermal drug delivery via synergistic action of chemicals. Biochim Biophys Acta, 2009; 1788:2362-73.

Khafagy el-S, Morishita M, Onuki Y, Takayama K. Current challenges in non-invasive insulin delivery systems: a comparative review. Adv Drug Deliv Rev, 2007; 59:1521-46.

Lavon I, Kost J. Ultrasound and transdermal drug delivery. Drug Discov Today, 2004; 9:670-6.

Lepore M, Pampanelli S, Fanelli C, Porcellati F, Bartocci L, Vincenzo $\mathrm{AD}$, et al. Pharmacokinetics and pharmacodynamics of subcutaneous injection of long-acting human insulin analog glargine, NPH insulin, and ultralente human insulin and continuous subcutaneous infusion of insulin lispro. Diabetes, 2000; 49:2142-8.

Li CY, Wang ZW, Tu C, Wang JB, Jiang BQ, Li Q, et al. Needle-free injection of insulin powder: delivery efficiency and skin irritation assessment. J Zhejiang UnivSci B, 2014; 15:888-99.

Mitragotri S, Kost J. Low-frequency sonophoresis: a review. Adv Drug Deliv Rev, 2004; 56:589-601.

Mohammadi-Samani S, Yousefi G, Mohammadi F, Ahmadi F. Meloxicam transdermal delivery: effect of eutectic point on the rate and extent of skin permeation. Iran J Basic Med Sci, 2014; 17:112-8.

Naik A, Kalia YN, Guy RH. Transdermal drug delivery: overcoming the skin's barrier function. Pharm Sci Technolo Today, 2000; 3:318-26.

Nielloud F, Marti G. 2000. Pharmaceutical Emulsions and Suspensions. France: CRC.

Ogura M, Paliwal S, Mitragotri S. Low-frequency sonophoresis: current status and future prospects. Adv Drug Deliv Rev, 2008; 60:121823.

Park EJ, Werner J, Smith NB. Ultrasound mediated transdermal insulin delivery in pigs using a lightweight transducer. Pharm Res, 2007; 24:1396-401.

Park EJ, Dodds J, Smith NB. Dose comparison of ultrasonic transdermal insulin delivery to subcutaneous insulin injection.Int $\mathrm{J}$ Nanomedicine, 2008; 3:335-41.

Polat BE, Blankschtein D, Langer R. Low-Frequency Sonophoresis: Application to the Transdermal Delivery of Macromolecules and Hydrophilic Drugs. Expert Opin Drug Deliv, 2010; 7:1415-32.

Saroha K., Sharma B, Yadav B. Sonophoresis: An advanced tool in transdermal drug delivery system. Int J Curr Pharm Res, 2011; 3: 89-97.

Sexton GB. Some fundamental principles in the treatment of skin disorders.Canadian medical association journal, 1940; 42:457-60.

Sintov AC, Wormser U. Topical iodine facilitates transdermal delivery of insulin. J Control Release, 2007; 118:185-8.

Wild S, Roglic G, Green A, Sicree R, King H. Global prevalence of diabetes: estimates for the year 2000 and projections for 2030. Diabetes Care, 2004; 27:1047-53.

\section{How to cite this article:}

Jabbari N, Mikaili P, Asghari MH, Ahmadin H. Eucerin impedes transdermal insulin delivery by sonophoresis in rats. J App Pharm Sci, 2016; 6 (10): 195-199. 